\title{
The effect of diagnostic amniocentesis and its complications on early spontaneous abortion
}

\author{
Fatemeh Tara ${ }^{1}$, Marzieh Lotfalizadeh ${ }^{1}$, Somayeh Moeindarbari ${ }^{2}$
}

\author{
${ }^{1}$ M.D., Associate Professor, Department of Obstetrics \& Gynecology, School of Medicine, Mashhad University of \\ Medical Sciences, Iran \\ ${ }^{2}$ M.D., Resident of Obstetrics \& Gynecology, Women's Health Research Center, Department of Obstetrics \& \\ Gynecology, School of Medicine, Mashhad University of Medical Sciences, Iran
}

\section{Type of article: Original}

\begin{abstract}
Introduction: The occurrence of early abortion after amniocentesis is a serious problem in the fields of obstetrics and gynecology, and it is always important to discover the factors influencing this phenomenon. The incidence rate has been reported in different studies, even up to about $10 \%$. So far, no studies have been conducted in Iran on the effect of amniocentesis and related complications on early abortion. The aim of this study was to determine the effects of amniocentesis and relevant complications on the incidence of early abortion in pregnant women undergoing amniocentesis.

Methods: This cohort study was conducted between March 2014 and March 2016 on pregnant candidates for amniocentesis referred to the perinatology clinic at Ommol-Banin Hospital, Mashhad, Iran. Amniocentesis was performed for all patients with about $20-30 \mathrm{cc}$ in the same manner by a perinatologist. Maternal blood group, causes of amniocentesis, amniotic fluid profile (liquid color), status of inserting the needle through the placenta during amniocentesis, amniotic fluid leakage, and bleeding after amniocentesis were considered as exposure factors, and spontaneous abortion after amniocentesis until the end of the 20th week of pregnancy was taken as a consequence. Data were analyzed using IBM-SPSS version 20 via t-test and chi-square. Relative risk (RR) was calculated to determine the causal relationship of exposure with the consequences of spontaneous abortion during the first week after amniocentesis.

Results: This study was performed on 1000 pregnant women with mean age of $33.4 \pm 6.0$ years (minimum 16, maximum 48 years). The incidence rate of spontaneous abortion after amniocentesis was obtained $1 \%$. There was no association among causes of amniocentesis, maternal blood group, maternal underlying diseases, history of diseases associated with pregnancy, and spontaneous abortion. Based on the chi-square test, a significant statistical relationship was found between amniotic fluid leakage and spontaneous abortion $(R R=15.37, p=0.001)$. There was also a significant statistical relationship between bleeding after amniocentesis and spontaneous abortion; so that in patients with more bleeding, spontaneous abortion was more prevalent $(\mathrm{RR}=6.83, \mathrm{P}=0.001)$.

Conclusion: According to the results, it seems that amniotic fluid leakage and bleeding after amniocentesis should be considered as two serious complications of amniocentesis, which can cause the incidence of spontaneous abortion in pregnant patients undergoing amniocentesis.
\end{abstract}

Keywords: Abortion, Amniocentesis, Complications, Pregnancy

\section{Introduction}

Amniocentesis means the extraction of amniotic fluid through a mother's abdominal walls and is the most commonly used method for detecting chromosomal abnormalities (1). This procedure is usually performed between the $15^{\text {th }}$ and $20^{\text {th }}$ week of pregnancy, and early measures can lead to less success, increased unsuccessful cell culture, higher risk, and fetal complications $(1,2)$. The occurrence of early abortion after amniocentesis is a serious problem in the fields of obstetrics and gynecology, and it is always important to discover the factors influencing this

\section{Corresponding author:}

Dr. Somayeh Moeindarbari, Women's Health Research Center, Imam Reza Hospital, Mashhad University of Medical Sciences, Mashhad, Iran. Tel: +98.5138022608, Email: moeins911@mums.ac.ir

Received: April 02, 2016, Accepted: July 23, 2016, Published: August 2016

iThenticate screening: July 13, 2016, English editing: July 28, 2016, Quality control: August 02, 2016

(c) 2016 The Authors. This is an open access article under the terms of the Creative Commons Attribution-NonCommercialNoDerivs License, which permits use and distribution in any medium, provided the original work is properly cited, the use is non-commercial and no modifications or adaptations are made. 
phenomenon $(3,4)$. The incidence rate has been reported in different studies, even up to about $10 \%(2-6)$. Amniocentesis is applicable for diagnostic and therapeutic measures, and the most diagnostic indications include prenatal genetic study, evaluation of fetal lung maturity, and assessment of fetal infection, anemia, determining the type of platelets or blood and neural tube defects (7-9). According to studies, bloody amniotic fluid occurs in less than $1 \%$ of amniocentesis, and the increased embryonic loss after bloody fluid has been reported in some studies; also, green or brown amniotic fluid has been associated with a higher rate of abortion in some studies (3, 9-11). In addition, other complications also have been shown, such as uterine contractions, temporary bleeding, loss of amniotic fluid, rupture of membrane, direct and indirect fetal damages as well as infection, which can all lead to abortion (12-14). However, no Iranian study has been conducted to investigate the effect of amniocentesis and related complications on early abortion with high statistical sample size; therefore, little information is available about this in Iran. By achieving a cause-effect relationship based on the results of the present study among the risk factors of early abortion after amniocentesis, the incidence rate of these adverse consequences can be reduced in the future by taking extra precautions. The aim of this study was to evaluate the effect of amniocentesis and its related complications on the incidence rate of early abortion in pregnant women undergoing amniocentesis.

\section{Material and Methods}

\subsection{Research design and setting}

This cohort study was conducted on pregnant patient candidates for amniocentesis referred to the perinatology clinic at Ommol-Banin Hospital, Mashhad, Iran, between March 2014 and March 2016.

\subsection{Sampling}

Considering alpha $=5 \%$, beta $=20 \%$, and $\mathrm{P}=10 \%$, the sample size was an estimated 1000 patients with the sample size formula based on prevalence of disease (14). All patients who were referred to our clinic during the study period were enrolled, and we used census method for sample selection.

\subsection{Selection criteria}

The inclusion criteria were 1) patients referred by a gynecologist due to abnormal biochemical or ultrasound markers detected during pregnancy; 2) history of anomaly in previous pregnancies or relatives such as Down's syndrome; 3 ) women having blood relations with their husbands. The exclusion criteria were missing in the followup period.

\subsection{Data collection}

First, patients' initial information was recorded such as age, gestational age based on the first day of the last menstrual period (LMP), body mass index (BMI), mother's blood group, underlying diseases and medical records in previous pregnancies, and referral indication for amniocentesis. Then an ultrasound was performed by the same experienced sonographer to determine fetus's living status, gestational age, the location of placenta, and the amount of amniotic fluid and to select the perfect spot for amniocentesis needle insertion. Before performing amniocentesis, the procedure and possible complications were explained to all patients plainly. Every step of amniocentesis was performed by an experienced perinatologist. First, the abdominal wall was disinfected. In an ultrasound-guided procedure, a needle number 23, manufactured by Dr.J (a Chinese company), was inserted into the skin of the mother's abdominal wall through the gestational sac to take 20-30 ml of amniotic fluid approximately. In cases that mothers visited the clinic after a gestational age of 16 weeks and 3 days, an extra $10 \mathrm{cc}$ of amniotic fluid was taken for an immediate test due to the time limitation on the abortion license. The patients were requested to visit the clinic in the presence of uterine cramps, hemorrhages, vaginal discharge, or fever signs during the first days after amniocentesis. Amniotic fluid color (bloody, transparent, green, or brown) and passage of the needle through placenta were also registered.

\subsection{Exposure and Outcome}

In this study, the factors of exposure included mothers' blood group, reason for amniocentesis, specifications of amniotic fluid (color), needle passing status through the placental in amniocentesis, amniotic fluid leakage, and spotting after amniocentesis. Factors of outcome were considered miscarriage after amniocentesis.

\subsection{Follow-up}

Patients were under weekly follow-ups on the phone until the 20th pregnancy week for the incidences of amniotic fluid leakage, spotting, and abortion. 


\subsection{Research ethics}

The procedure of amniocentesis and possible complications were explained to patients before conducting the study. All the patients filled out the consent form, indicating that they participated in the research plan consciously. This research plan was approved by the Ethics Committee of Mashhad University of Medical Sciences.

\subsection{Statistical analyses}

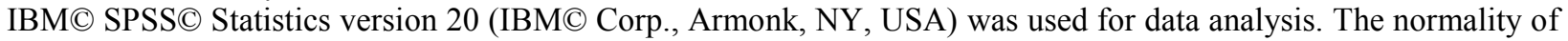
data was first determined with the Kolmogorov-Smirnov test. If data were distributed normally, the t-test was used to determine the relationship between the mean of a quantitative variables in two groups with positive and negative outcomes. The U-Mann-Whitney test was conducted for non-normally distributed data. The chi-squared test was employed to determine the relationship between qualitative variables with positive and negative outcomes. Given the fact that this was a cohort study, the relative risk (RR) was calculated in order to determine the cause-effect relationship between exposures and outcomes of miscarriage during one week after amniocentesis. If RR was equal to 1 , the exposure would not be related to the outcome. If it was greater or smaller than 1 , it would be related to the final outcome.

\section{Results}

\subsection{Baseline characteristics}

During the study, 2213 pregnant women, who were candidates for amniocentesis, were referred to our clinic among whom 1032 patients were included. Later on, 32 of them were excluded from the study due to missing the followup. Finally, the study was carried out on 1000 pregnant women with the average age of $33.4 \pm 6.0$ years (minimum of 16 and maximum of 48 years) and with the average BMI of $26.1 \pm 4.1 \mathrm{~kg} / \mathrm{m} 2$.

\subsection{Incidence of spontaneous abortion}

All patients underwent amniocentesis, and the follow-up after amniocentesis revealed that the total incidence of spontaneous abortion after amniocentesis was $1 \%$. Based on the outcome (whether spontaneous abortion occurred during the following amniocentesis or not), the pregnant women were placed into two groups: the group with spontaneous abortion (10 pregnant women) and the group without it (990 pregnant women). There were no statistically significant differences between the groups with respect to the quantitative data of age, age of pregnancy, and average BMI (Table 1).

Table 1. Comparison of the two groups with and without spontaneous abortions following amniocentesis with respect to the characteristics of the pregnant women.

\begin{tabular}{|c|c|c|c|c|}
\hline \multicolumn{2}{|l|}{ Variable, unit } & $\begin{array}{l}\text { Spontaneous } \\
\text { abortion }(n=10)\end{array}$ & $\begin{array}{l}\text { No spontaneous } \\
\text { abortion }(n=990)\end{array}$ & $\begin{array}{l}p \text { - } \\
\text { value }\end{array}$ \\
\hline \multicolumn{2}{|c|}{ Average age of pregnant women (years) } & $33.47 \pm 6.12$ & $32.12 \pm 6.28$ & 0.401 \\
\hline \multicolumn{2}{|c|}{ Average age of pregnancy (weeks) } & $16.35 \pm 3.31$ & $15.81 \pm 4.19$ & 0.339 \\
\hline \multicolumn{2}{|l|}{ BMI $\left(\mathrm{kg} / \mathrm{m}^{2}\right)$} & $26.17 \pm 4.34$ & $25.83 \pm 5.28$ & 0.721 \\
\hline \multirow[t]{3}{*}{$\begin{array}{l}\text { Amniocentesis indications, } \\
n(\%)\end{array}$} & $\begin{array}{l}\text { Disorders detected in } \\
\text { screening by ultrasound }\end{array}$ & $2(20)$ & $43(4.5)$ & \multirow[t]{3}{*}{0.810} \\
\hline & $\begin{array}{l}\text { Disorders detected in } \\
\text { screening by biochemical } \\
\text { markers }\end{array}$ & $5(50)$ & $570(58)$ & \\
\hline & Other causes & $3(30)$ & $337(38.1)$ & \\
\hline \multirow{4}{*}{$\begin{array}{l}\text { Blood groups of pregnant } \\
\text { women, } n(\%)\end{array}$} & A & $4(40)$ & $338(34.1)$ & \multirow[t]{4}{*}{0.394} \\
\hline & $\mathrm{AB}$ & $1(10)$ & $119(12.0)$ & \\
\hline & $\mathrm{O}$ & $3(30)$ & $293(29.5)$ & \\
\hline & $\mathrm{B}$ & $2(20)$ & $240(24.2)$ & \\
\hline \multirow{2}{*}{$\begin{array}{l}\text { History of diseases in the } \\
\text { pregnant women, } n(\%)\end{array}$} & Diabetes & $1(10)$ & $60(6.0)$ & \multirow[t]{2}{*}{0.500} \\
\hline & Preeclampsia & $1(10)$ & $45(4.5)$ & \\
\hline
\end{tabular}

\subsection{Background status of pregnant women and spontaneous abortion}

Results indicated no relationship among the reasons for performing amniocentesis, blood groups, background diseases, history of diseases related to pregnancy, and spontaneous abortion (Table 1). 


\subsection{Fetal screening and spontaneous abortion}

Based on results of this study, there was no statistically significant relationship among disorders detected in screening by ultrasound or by biochemical markers and spontaneous abortion following amniocentesis (Table 1).

\subsection{Characteristics of the amniotic fluid and spontaneous abortion}

Amniocentesis was performed in every patient on the first attempt. In 94 patients $(9.4 \%)$, the needle passed through the placenta during amniocentesis; in 42 patients (4.2\%), the amniotic fluid drawn out of the mother's abdomen was bloody. There were no statistically significant relationship between passing the needle through the placenta or the color of the amniotic fluid and spontaneous abortion (Table 2).

Table 2. Comparison of the groups with and without spontaneous abortions following amniocentesis based on features of amniocentesis and on its complications.

\begin{tabular}{|l|l|l|l|l|}
\hline Variable & $\begin{array}{l}\text { Spontaneous abortion, } \\
(n=10)\end{array}$ & $\begin{array}{l}\text { No spontaneous } \\
\text { abortions, }(n=990)\end{array}$ & $\begin{array}{l}p \text { - } \\
\text { value }\end{array}$ \\
\hline Passage of needle through the placenta, number $(\%)$ & $0(0)$ & $49(4.9)$ & 0.999 \\
\hline $\begin{array}{l}\text { Color of the amniotic fluid, } n \\
(\%)\end{array}$ & Bloody & $1(10)$ & $32(3.2)$ & 0.201 \\
\cline { 2 - 5 } & Opaque or brown & $0(0)$ & $7(0.7)$ & 0.999 \\
\hline $\begin{array}{l}\text { Other complications following } \\
\text { amniocentesis }\end{array}$ & $\begin{array}{l}\text { Leakage of } \\
\text { amniotic fluid }\end{array}$ & $2(20)$ & $14(1.4)$ & 0.001 \\
\cline { 2 - 5 } & Spotting & $1(10)$ & $15(1.5)$ & 0.031 \\
\hline
\end{tabular}

\subsection{Short-term complications following amniocentesis}

Leakage of amniotic fluid was observed in 16 patients (1.6\%). In all such cases, the leakage started immediately after amniocentesis up to 24 hours after and stopped during the one-week follow-up. Moreover, spotting was also observed in 16 patients $(1.6 \%)$ following amniocentesis. It started from 24 to 72 hours after amniocentesis and stopped during the one-week follow-up. There were two statistically significant relationships following amniocentesis between leakage of amniotic fluid and spontaneous abortion $(R R=15.37, p=0.001)$. Moreover, a significant relationship was observed between spotting and spontaneous abortion $(\mathrm{p}=0.001, \mathrm{RR}=6.83)$ (Table 2$)$.

\section{Discussion}

Amniocentesis is a procedure in which amniotic fluid is extracted through the amniotic sac and is the most common method to detect genetic disorders of the fetus $(2,3,5)$. If the procedure is performed early, it would have a lower success rate, and the cell culture would be unsuccessful. It also causes several fetal complications and risks $(2,5)$. In the present study, amniocentesis was performed on pregnant women with gestational age of less than 20 weeks. In cases in which the visit was after the gestational age of 16 weeks and 3 days, due to the time limit allowed for abortion, $10 \mathrm{ml}$ extra of amniotic fluid was taken for immediate testing, and patients were followed-up weekly until the end of the 20th week of pregnancy. In this study, the relationship between the incidence of abortion following amniocentesis and the passage of needle through the placenta during amniocentesis was examined, for which statistical analysis showed no significant relationship. In spontaneous abortion cases, needles had not passed the placenta, while needles had passed the placenta in $4.9 \%$ of the cases without spontaneous abortion, which might be due to the postion of placenta. However, Anderson (1989) stated that the position of placenta has no effect on the incidence of spontaneous abortion (15). Giorlando (1994) examined the amniocenteses that were performed through passing the placenta. In 1487 cases, the needle had passed anterior placenta; in 3077 cases, the needle had directly entered the amniotic fluid without passing the placenta. Patients were followed-up for 30 days after the amniocentesis (16). Two cases of spontaneous abortion were reported in the group with passage of the needle through placenta, and five cases of spontaneous abortion were reported in cases without passage of the needle through placenta. It was suggested that the risk of spontaneous abortion was similar in both groups. Martin (1997) reported no difference in risk of spontaneous abortion in anterior or posterior placenta cases and suggested that passage of the needle does not increase the risk of spontaneous abortion (17). In a study by Bombard (1995), placenta was anterior in 518 cases, and the needle had passed through the placenta in 306 amniocentesis cases. Spontaneous abortion following passage of the needle through placenta occurred within an average of 26 days after amniocentesis. The rate of fetal loss was similar in both groups and equal to $1.9 \%$ (18). Crane (1984) and Kang (2006) reported increased incidence of spontaneous abortion in cases where the needle had passed through the placenta $(11,19)$. In the present study, we investigated the relationship between the incidence of spontaneous abortion following amniocentesis and the amniotic fluid being bloody, non-clear, or brown. The amniotic fluid was bloody in $10 \%$ in the spontaneous abortion cases and in $3.2 \%$ of the cases without spontaneous abortion. The 
statistical analysis showed increased incidence of spontaneous abortion in cases with bloody amniotic fluid. No case of brown or non-clear amniotic fluid was observed in the spontaneous abortion cases. Kappel (1987) reported an increased spontaneous abortion risk when the amniotic fluid is bloody. According to Taber (1986), non-clear amniotic fluid is associated with an increased risk of spontaneous abortion $(20,21)$. Hess (1986) examined the effect of abnormal color of amniotic fluid obtained from amniocentesis in 7018 subjects. The amniotic fluid color was abnormal in $1.4 \%$ of the cases, which increased the incidence of spontaneous abortion by $7 \%$ compared with the control group (22). In this study, we examined the relationship between the incidence of spotting and leakage of amniotic fluid following amniocentesis and the incidence of spontaneous abortion. In $22.2 \%$ of the cases with spontaneous abortion and in $1.2 \%$ of the cases without spontaneous abortion, amniotic fluid leakage occurred within the first 10 days after amniocentesis. Statistical analysis showed that leakage of amniotic fluid following amniocentesis increases the risk of spontaneous abortion. In $16.7 \%$ of the cases with spontaneous abortion and in $1.3 \%$ of the cases without spontaneous abortion, spotting occurred within the first 10 days after the amniocentesis. Statistical analysis showed that spotting following amniocentesis increases the risk of spontaneous abortion. Anderson (1989) and Koralo (2012) reported that history of bleeding in pregnancy is associated with an increased risk of spontaneous abortion following amniocentesis $(15,23)$. Maternal age and gestational age were other unrelated variables in our study, while a study conducted in 2011 showed that both variables are involved in spontaneous abortion before the gestational age of 24-28 weeks and the maternal age of 41 years. In the present study, maternal age, underlying and pregnancy-related disease, blood type, and body mass index were not related to spontaneous abortion following amniocentesis. As mentioned earlier, the only two factors of amniotic fluid leakage and spotting were causes of spontaneous abortion following amniocentesis. Amniotic fluid leakage was observed in $1.6 \%$ of the cases. In all patients, amniotic fluid leakage started after 24 hours after amniocentesis and ended within a week of follow-ups. Spotting was observed in $1.6 \%$ of cases following amniocentesis and between 24 and 72 hours after amniocentesis. It discontinued in all patients during the one-week follow-up. A statistically significant relationship was found between amniotic fluid leakage and spotting following amniocentesis and spontaneous abortion. The incidence of amniotic fluid leakage and spotting was higher in patients with spontaneous abortion. One of the major limitations of this study is in regards to its one center study.

\section{Conclusions}

According to the results, it seems that maternal baseline characteristics such as age, age of pregnancy, BMI, indication of amniocentesis, blood group, and disease history might not have a relationship with spontaneous abortion after amniocentesis. However, among the post-amniocentesis complications, leakage of amniotic fluid and spotting were two important factors that have been affecting spontaneous abortion. By the way, more observational studies are needed for confirmation of our study results.

\section{Acknowledgments:}

Hereby researchers consider it necessary to appreciate Mashhad University of Medical Sciences Deputy of Research and Technology for financial support of this project. This manuscript was derived from Obstetrics and Gynecology Residency thesis of Somayeh Moeindarbari (M.D.) with the registration code of 930253.

\section{Conflict of Interest:}

There is no conflict of interest to be declared.

\section{Authors' contributions:}

All authors contributed to this project and article equally. All authors read and approved the final manuscript.

\section{References:}

1) Rapp R. Testing women, testing the fetus: The social impact of amniocentesis in America: Routledge; 2004.

2) Enzensberger C, Pulvermacher C, Degenhardt J, Kawacki A, Germer U, Gembruch U, et al. Fetal loss rate and associated risk factors after amniocentesis, chorionic villus sampling and fetal blood sampling. Ultraschall Med. 2012; 33(7): 75-9. doi: 10.1055/s-0031-1299388. PMID: 22623130.

3) Bakker M, Birnie E, Robles de Medina P, Sollie K, Pajkrt E, Bilardo CM. Total pregnancy loss after chorionic villus sampling and amniocentesis - A cohort study. Ultrasound Obstet Gynecol. 2016. doi: 10.1002/uog.15986. PMID: 27255564.

4) Kollmann M, Haeusler M, Haas J, Csapo B, Lang U, Klaritsch P. Procedure-related complications after genetic amniocentesis and chorionic villus sampling. Ultraschall Med. 2012; 34(4): 345-8. doi: 10.1055/s0032-1312939. PMID: 22723040. 
5) Enzensberger C, Pulvermacher C, Degenhardt J, Kawecki A, Germer U, Weichert J, et al. Outcome after second-trimester amniocentesis and first-trimester chorionic villus sampling for prenatal diagnosis in multiple gestations. Ultraschall Med. 2014; 35(2): 166-72. doi: 10.1055/s-0032-1330700. PMID: 23696061.

6) Pruggmayer M, Baumann P, Schütte H, Osmers R, Bartels I, Jovanovich V, et al. Incidence of abortion after genetic amniocentesis in twin pregnancies. Prenat Diagn. 1991; 11(8): 637-40. doi: 10.1002/pd.1970110822. PMID: 1766936.

7) Lehmann LS. How can we improve amniocentesis decision-making? Isr J Health Policy Res. 2016 ; 5: 4. doi: 10.1186/s13584-016-0060-0. PMID: 26855767, PMCID: PMC4743405.

8) Prefumo F, Jauniaux E. Amniocentesis for fetal karyotyping: the end of an era? BJOG. 2016; $123(1)$ : 99. doi: 10.1111/1471-0528.13497. PMID: 26715343.

9) Theodora M, Antsaklis A, Antsaklis P, Blanas K, Daskalakis G, Sindos M, et al. Fetal loss following second trimester amniocentesis. Who is at greater risk? How to counsel pregnant women? J Matern Fetal Neonatal Med. 2016; 29(4): 590-5. doi: 10.3109/14767058.2015.1012061. PMID: 25747948.

10) Palle C, Andersen JW, Tabor A, Lauritsen JG, Bang J, Philip J. Increased risk of abortion after genetic amniocentesis in twin pregnancies. Prenatal diagnosis. 1983; 3(2): 83-9. doi: 10.1002/pd.1970030202. PMID: 6622400 .

11) Kong CW, Leung TN, Leung TY, Chan LW, Sahota DS, Fung TY, et al. Risk factors for procedure related fetal losses after mid - trimester genetic amniocentesis. Prenat Diagn. 2006; 26(10): 925-30. doi: 10.1002/pd.1528. PMID: 16838383.

12) Saltvedt $S$, Almström H. Fetal loss rate after second trimester amniocentesis at different gestational age. Acta Obstet Gynecol Scand. 1999; 78(1): 10-4. doi: 10.1080/j.1600-0412.1999.780104.x. PMID: 9926885.

13) Kan AS, Lee CP, Leung KY, Chan BC, Tang MH, Chan VH. Outcome of twin pregnancies after amniocentesis. J Obstet Gynaecol Res. 2012; 38(2): 376-82. doi: 10.1111/j.1447-0756.2011.01721.x. PMID: 22229750.

14) Theodora M, Antsaklis A, Antsaklis P, Blanas K, Daskalakis G, Sindos M, et al. Fetal loss following second trimester amniocentesis. Who is at greater risk? How to counsel pregnant women? J Matern Fetal Neonatal Med. 2016; 29(4): 590-5. doi: 10.3109/14767058.2015.1012061. PMID: 25747948.

15) Andreasen E, Kristoffersen K. Incidence of spontaneous abortion after amniocentesis: influence of placental localization and past obstetric and gynecologic history. Am J Perinatol. 1989; 6(2): 268-73. doi: 10.1055/s-2007-999589. PMID: 2653338.

16) Giorlandino C, Mobili L, Bilancioni E, D'Alessio P, Carcioppolo O, Gentili P, et al. Transplacental amniocentesis: is it really a higher-risk procedure? Prenat Diagn. 1994; 14(9): 803-6. doi: 10.1002/pd.1970140907. PMID: 7845887.

17) Marthin T, Liedgren S, Hammar M. Transplacental needle passage and other risk-factors associated with second trimester amniocentesis. Acta Obstet Gynecol Scand. 1997; 76(8): 728-32. doi: 10.3109/00016349709024337. PMID: 9348248.

18) Bombard AT, Powers JF, Carter S, Schwartz A, Nitowsky HM. Procedure-related fetal losses in transplacental versus nontransplacental genetic amniocentesis. Am J Obstet Gynecol. 1995; 172(3): 868-72. doi: 10.1016/0002-9378(95)90013-6. PMID: 7892877.

19) Crane JP, Kopta MM. Genetic amniocentesis: Impact of placental position upon the risk or pregnancy loss. Am J Obstet Gynecol. 1984; 150(7): 813-6. doi: 10.1016/0002-9378(84)90454-X. PMID: 6391172.

20) Tabor A, Philip J, Madsen M, Bang J, Obel EB, Nørgaard-Pedersen B. Randomised controlled trial of genetic amniocentesis in 4606 low-risk women. Lancet. 1986; 1(8493): 1287-93. doi: 10.1016/S01406736(86)91218-3. PMID: 2423826.

21) Kappel B, Nielsen J, Brogaard Hansen K, Mikkelsen M, Therkelsen AJ. Spontaneous abortion following mid - trimester amniocentesis. Clinical significance of placental perforation and blood - stained amniotic fluid. Br J Obstet Gynaecol. 1987; 94(1): 50-4. doi: 10.1111/j.1471-0528.1987.tb02252.x. PMID: 3814555.

22) Hess LW, Anderson RL, Golbus MS. Significance of opaque discolored amniotic fluid at second-trimester amniocentesis. Obstet Gynecol. 1986; 67(1): 44-6. PMID: 3940336.

23) Wapner RJ, Johnson A, Davis G, Urban A, Morgan P, Jackson L. Prenatal diagnosis in twin gestations: a comparison between second-trimester amniocentesis and first-trimester chorionic villus sampling. Obstet Gynecol. 1993; 82(1): 49-56. PMID: 8515925. 